\section{Alteraciones osteoarticulares en el músico adulto mayor de instrumentos viento-madera}

\section{Osteoarticular alterations in the elderly musician of Wind-Wood Instruments}

\author{
Salomé Hernández Félix ${ }^{1}$ \\ María del Pino Quintana Montesdeoca² \\ Francisco José Hernández Martínez ${ }^{3, *}$ \\ Salomé Félix Mateu ${ }^{4}$ \\ Bienvenida del Carmen Rodríguez de Vera ${ }^{5}$ \\ Juan Fernando Jiménez Díaz
}
1. Odontóloga. Facultad de Odontología. Universitat de València. Valencia. España.
2. Departamento de Matemáticas. Universidad de Las Palmas de Gran Canaria. Islas Canarias. España.
3. Departamento de Enfermería del Trabajo. Cabildo de Lanzarote. Islas Canarias. España.
4. Músico. Conservatorio Insular de Música de Lanzarote. Islas Canarias. España.
5. Departamento de Enfermería. Universidad de Las Palmas de Gran Canaria. Islas Canarias. España.

${ }^{*}$ Autor para correspondencia.

Correo electrónico: francisco.hernandezmartinez@ulpgc.es (Fco. José Hernández Martínez).

Recibido el 23 de octubre de 2019: aceptado el 5 de noviembre de 2019.

\section{RESUMEN}

Objetivo: Valorar si existen cambios a nivel bucodental en adultos mayores, instrumentistas de viento-madera por el uso del instrumento a lo largo de más de 35 años de profesión y compararlos con un grupo control. Metodología: Estudio de tipo observacional, descriptivo de corte transversal, entre músicos profesionales, adultos mayores, de 60 ańos o más, con más de 35 ańos de profesión y que toquen instrumentos de "viento-madera". La muestra inicial de estudio estaba formada por 57 músicos. El instrumento de recogida de datos utilizado se ha elaborado de forma exclusiva. El análisis estadístico se llevó a cabo mediante SPSS Statistics 23.0. Resultados: Media de edad $63 \pm 1,28$ años, horas de estudio diario $3,47 \pm 3,3$ horas con experiencia profesional de 40,12 $\pm 12,98$ ańos. Dependiendo del tipo de instrumento, embocadura, años de profesión y las fuerzas ejercidas por la lengua, labios, así como las de oclusión dental, aparecen distintas alteraciones en la articulación temporomandibular de los músicos, variaciones en el aparato estomatognático en músicos con instrumentos de lengüeta tipo bisel y maloclusiones junto a desgaste de los bordes incisales en los de lengüeta simple y doble. Es significativa la aparición de trastornos de la articulación temporomandibular entre instrumentistas de viento-madera y el grupo control $(p=0,0236)$. Conclusiones: Según el tipo de instrumento de viento y del contacto de la "boquilla" con el sistema estomatognático, este estudio ha demostrado que tocar un instrumento de viento-madera a nivel profesional durante más de 35 años afecta a la posición de los dientes y donde las horas de estudio y las fuerzas continuas e intermitentes necesarias para tocar son factores etiológicos predisponentes de maloclusión y de la aparición de alteraciones osteoarticulares en la cavidad oral de los músicos adultos mayores.

PALABRAS CLAVE: Músico adulto mayor, alteraciones osteoarticulares, fuerzas de presión, instrumento musical.

\section{ABSTRACT}

Objectives: To assess whether there are changes at the oral level in older adults, woodwind players for the use of the instrument over more than 35 years of profession and compare them with a control group. Methodology: Observational, descriptive cross-sectional study, among professional musicians, seniors, 60 years of age or older, with more than 35 years of profession and playing "woodwind" instruments. The initial study sample was formed by 57 musicians. The data collection instrument used has been developed exclusively. The statistical analysis SPSS Statistics 23.0. Results: Mean age $63 \pm 1.28$ years, daily study hours $3.47 \pm 3.3$ hours with professional experience of $40.12 \pm 2.98$ years. Depending on the type of instrument, mouthpiece, years of profession and the forces exerted by the tongue, lips, as well as those of dental occlusion, different alterations in the Temporomandibular Joint of musicians appear variations in the stomatognathic apparatus in musicians with type tongue instruments bevel and malocclusions with wear of the incisal edges in the single and double tongue. The appearance of temporomandibular joint disorders between woodwind players and the control group is significant $(\mathrm{p}=0.0236)$. Conclusions: Depending on the type of wind instrument and the contact of the "mouthpiece" with the stomatognathic system, this study has shown that playing a professional wind-wood instrument for more than 35 years affects the position of the teeth and where the hours of study and the continuous and intermittent forces necessary to play; they are predisposing etiological factors of malocclusion and the appearance of osteoarticular alterations in the oral cavity of older adult musicians.

KEYWORDS: Older musician, osteoarticular alterations, pressure forces, musical instrument.

\section{- INTRODUCCIÓN}

La mayoría de la gente no asocia la música con el esfuerzo quizás porque no vemos la música como un trabajo. Lo cierto es que detrás del espectáculo hay un mundo de disciplina, de ensayos, de horas de estudio y todo delante de la mirada crítica del público. Con mucha frecuencia ni los artistas ni los profesionales de la salud son conscientes del esfuerzo físico y psíquico que implica tocar un instrumento. Parece ser que existen motivos diferentes que favorecen la etiología de estos problemas; entre ellos, el tipo de instrumento que se utiliza (forma, diseńo, peso, 
longitud, etc.), ya que tienen una forma estandarizada, con la finalidad de conseguir una calidad determinada, sobre todo con respecto a la interpretación y el sonido. También es muy importante el esfuerzo para conseguir notas muy graves y/o agudas, y no hay que olvidar la duración de la partitura o el tipo de música que se interpreta.

En todos los instrumentistas, y sobre todo en los de viento, la gesticulación interpretativa modifica constantemente la presión que soporta la musculatura oral y el aparato estomatognático y se realizan movimientos repetitivos o posiciones forzadas de la mandíbula que pueden desencadenar alteraciones y trastornos anatomofisiológicos.

Otros factores que también hay que tener en cuenta son: la edad con la que comienzan a estudiar, el sexo del instrumentista y las características anatómicas o fisiológicas de cada persona (manos pequeñas, boca pequeña, labios gruesos, etc. $)^{1-6}$.

En los músicos de la familia de viento hay una relación entre el sonido y los dientes, ya que estos dan apoyo a los labios, mejillas y lengua, determinando la producción de sonido. Todo el complejo de estructuras anatómicas alrededor de la boca y la forma en que se usan para tocar el instrumento de viento se llama "embocadura". Por tanto, los tres componentes principales que afectan a la embocadura de un músico son la lengua, los dientes y los músculos de las mejillas y los labios ${ }^{7-8}$.

Debido a esta relación, los músicos pueden sufrir problemas relacionados con la salud oral, sobre todo aquellos que se dedican a tocar instrumentos de viento ${ }^{6,8-9}$

Existen dos familias en los instrumentos de viento: los instrumentos de viento-metal y los instrumentos de viento-madera, y se diferencian por cómo se produce el sonido.

En los instrumentos de viento-madera, la boquilla se coloca entre los labios, mientras que los instrumentos de viento-metal tienen una boquilla que se coloca contra los labios, para producir la vibración responsable del sonido. En la práctica interpretativa de estos instrumentos de viento, el sonido producido depende del propio instrumento, del instrumentista en sí y de las boquillas, cañas o lengüetas utilizadas por cada instrumentista ${ }^{6,10}$.

Los músicos deben emplear años y horas de estudio para adaptar el instrumento a la boca e intentar adaptarse a la embocadura del instrumento, y han de mantener posturas durante largos periodos, las cuales generan sobrecargas en órganos y aparatos que no están diseńados para soportar este esfuerzo continuado. Numerosos son los estudios publicados desde finales de los años 90 del pasado siglo hasta la actualidad que hacen referencia a que la práctica continuada con instrumentos de viento puede causar o desencadenar numerosos problemas en la salud oral de los músicos, ya que la forma de la boca influirá muchísimo en el modo de tocar un instrumento de viento. También el propio instrumento puede afectar a la morfología de la boca, pudiendo inducir y provocar malocusiones ${ }^{11-13}$, resaltando varios autores que estos individuos están predispuestos a sufrir y desarrollar este tipo de patología y anomalías por influencia de las horas de ensayo, dedicación y por el estrés en la búsqueda de la perfección de la técnica interpretativa ${ }^{4,8,14}$.

En el envejecimiento, todos los órganos y sistemas del cuerpo humano sufren cambios morfológicos y funcionales, donde también se ve afectada la cavidad bucal, conociéndose que un número importante de ancianos presenta enfermedades en los tejidos de sostén y en el aparato estomatognático en genera ${ }^{15}$. Misericordia García, junto a las enfermeras Noriega y Torres ${ }^{16}$, describen que en la boca de los adultos mayores se produce una disminución de la producción de saliva, erosión de dentina y esmalte, reabsorción de la raíz y migración apical de estructuras de soporte del diente.

El periodonto puede reaccionar al proceso de envejecimiento de dos formas: si existe poca higiene bucal, el acúmulo de placas dentobacte- rianas provoca gingivitis y, con el tiempo, en los pacientes susceptibles, retracción gingival a nivel de cuello del diente, descubriendo el cemento y, por lo tanto, disminuyendo la resistencia a las caries. En las encías ocurre la pérdida del punteado, aspecto fibroso, delgadez o falta de la capa queratinizada. En este sentido, los cambios bucales relacionados con la edad pueden tener dos orígenes: como expresión del envejecimiento propiamente dicho o como consecuencia de factores internos fisiológicos que no provocan enfermedad, pero inducen cambios bioquímicos funcionales y estructurales ${ }^{15,17}$.

Si consideramos el sexo en el adulto mayor, Ortega Velazco y sus colaboradores ${ }^{18}$ plantean que en las mujeres se nota más la reducción de la velocidad del flujo salival después de la menopausia y la reabsorción ósea también es mayor por el adelgazamiento e incremento de la porosidad entre los 40 y los 80 ańos.

En cuanto a estas maloclusiones, se pueden observar, dependiendo del tipo de embocadura del instrumento, una mayor tendencia al retrognatismo mandibular y un resalte aumentado (en instrumentos de lengüeta simple como el clarinete o el saxofón) o por el contrario una disminución del resalte y aumento de la sobremordida en otros instrumentos como los de lengüeta doble o bisel y viento metal $^{9,11}$.

En realidad, no existe la embocadura ideal y esta se conforma a través de la práctica, día tras día, por lo que la fisiología de nuestro esqueleto dentofacial condicionará el desarrollo y el uso de nuestra musculatura y como no, el lugar donde se colocará la boquilla del instrumento de viento. En esta actividad también tiene un papel muy importante la respiración, ya que es necesario tener un gran dominio sobre ella.

Debemos ańadir que las edades tempranas a las que los músicos comienzan a tocar el instrumento condicionan incluso el desarrollo fisiológico, por lo que la relación se vuelve bidireccional: nuestra fisiología condiciona nuestra embocadura, pero la práctica de dicha embocadura también acaba condicionando la fisiología ${ }^{8}$. En estos últimos años ha sido tan importante el diagnóstico de las patologías asociadas al aparato estomatognático en los músicos, que se ha llegado a desarrollar programas y estudios de ingeniería industrial para diseñar instrumentos que sean capaces de detectar los esfuerzos labiales, la fuerza máxima y la resistencia que ejercen estos sobre la musculatura de la boca, maxilares y dientes. Como ejemplo, cabe destacar que un clarinetista genera, al tocar el instrumento, entre 800 y 1000 microdeformaciones a nivel del sistema estomatognático ${ }^{19,20}$.

\section{- CARACTERÍSTICAS DE LOS INSTRUMENTOS ESTUDIADOS EN ESTE TRABAJO}

Clarinete: está formado por una boquilla donde se inserta la caña de lengüeta simple, un barrilete, cuerpo superior, inferior y campana. Frecuentemente son de madera noble, aunque pueden fabricarse de otros materiales como el plástico que usualmente se utilizan para tocar por la calle.

Flauta: está formada por la boquilla o cabeza (que contiene un bisel), el cuerpo principal con la mayor parte de las teclas y el pie con la llave última para el dedo meñique derecho.

Fagot: está formado por un cono de 2,5 m curvado. En el extremo superior tiene una pieza de metal curva, que es la boquilla donde se inserta la cańa doble.

Oboe: formado por tres partes: cabeza o cuerpo superior y donde se introduce la cańa por una pequeńa apertura, el cuerpo inferior y la campana. Todas encajan con una especie de corcho y su lengüeta es doble $e^{21,22}$ 


\section{- CARACTERÍSTICA DE CADA EMBOCADURA}

Bisel: a este grupo pertenece la flauta. El instrumentista, para realizar la correcta embocadura, debe colocar la boquilla del bisel sobre el labio inferior y el labio superior es estirado hacia abajo hasta formar una pequeña abertura; al aumentar o disminuir la tensión que ejerce el labio superior, se producen diferentes tonos.

Lengüeta simple: a este grupo pertenece el clarinete y el saxofón. Tienen una cańa de madera que se une con una abrazadera a la boquilla. El labio inferior envuelve los dientes anteroinferiores, sobre el cual descansa la mayor parte del peso del instrumento. La boquilla es mantenida firme con una ligera presión de los dientes anterosuperiores, y el labio superior cierra la boquilla de forma hermética. La principal diferencia entre la boquilla de saxofón y la del clarinete es que la boquilla de saxofón debe entrar en la boca en un ángulo mucho más inferior o plano que la del clarinete ${ }^{23,24}$.

Lengüeta doble: a este grupo pertenecen el oboe, el corno inglés y el fagot. En la embocadura de estos instrumentos tanto el labio superior como el inferior envuelven los bordes incisales de los incisivos superiores e inferiores, formando cada uno un lecho donde se coloca la boquilla del instrumento ${ }^{25}$.

\section{- OBJETIVOS}

Valorar los cambios a nivel bucodental en los adultos mayores, instrumentistas de viento-madera, y compararlos con un grupo control de músicos, también adultos mayores, donde el instrumento no tiene relación con el aparato estomatognático.

\section{- METODOLOGÍA}

Tipo de estudio: estudio observacional, descriptivo de corte transversal.

Población de estudio: músicos profesionales, adultos mayores, de 60 ańos o más, con más de 35 años de profesión y que toquen instrumentos de "viento-madera". La muestra inicial de estudio estaba formada por 57 músicos. Aplicando los criterios de exclusión, la muestra total que conforma el estudio corresponde a 12 músicos mayores de 60 años y con experiencia como profesionales de más de 35 años, con un promedio de al menos 3 horas diarias de estudio y que no toquen instrumentos con diferentes lengüetas.

En cuanto a los criterios de exclusión del estudio, tenemos que destacar que no formarán parte de este los músicos que han sido portadores de ortodoncias con anterioridad, que no tengan dientes supernumerarios, ni les falten piezas dentarias.

El instrumento de recogida de datos utilizado en este estudio se ha elaborado de forma exclusiva para el mismo. Se trata de un cuestionario de carácter anónimo y consta de dos apartados. Un documento donde se recogen datos sociodemográficos del músico (edad, sexo, etc.), datos relativos al instrumento, así como al uso de este, y una parte dedicada a cuestiones relacionadas con la oclusión del individuo. El segundo documento se corresponderá a un dosier de fotografías de la boca y dientes de los músicos (cuyas fotos recogerán única y exclusivamente esa parte de la cara para salvaguardar aspectos relacionados con la intimidad).

El periodo de recogida de datos se realizó entre enero y marzo de 2019 en orquestas y bandas de música profesionales.

El análisis estadístico de las variables del estudio se realizó con el paquete estadístico SPSS Statistics 23.0. Se consideró significación estadística cuando $p<0,05$.
En todo momento se tuvo en cuenta las distintas concepciones éticas, creencias y opiniones de los músicos adultos encuestados. El estudio contó con la aprobación del Comité de Ética de la Universidad de Valencia. En cuanto a los cuestionarios, tal como hemos indicado en los párrafos anteriores, son anónimos para preservar la intimidad y libertad del encuestado, respetando así su derecho a la confidencialidad. A todos los sujetos participantes en el estudio se les entregó un consentimiento informado que cumple los criterios definidos en la LOPD 15/1999 y se les explicó la posibilidad de ejercer los derechos ARCO (acceso, rectificación, cancelación u oposición).

\section{- RESULTADOS}

La muestra del estudio $(n=12)$, según el tipo de embocadura e instrumento, está compuesta por: 4 flautistas, 4 clarinetistas, 2 oboes y $2 \mathrm{fa}$ gotes. Respecto al sexo, se ha estudiado una muestra paritaria, con igual número de hombres y mujeres que tocan el mismo tipo de instrumento. Cabe destacar que no hay diferencias significativas entre el sexo, la edad y el tipo de instrumento, así como tampoco es significativo el tipo de alteración a nivel del aparato estomatognático con el género.

La media de edad es de $63 \pm 1,28$ ańos, con una moda de 62 años, una media de horas de estudio diario de $3,47 \pm 3,3$ horas y una experiencia profesional como músico instrumentista de $40,12 \pm 2,98$ años.

El 50\% de la muestra tiene 62 ańos, con experiencia profesional de más de 35 ańos y toca una media entre 3 y 5 horas al día. El profesional de menor edad se corresponde con una mujer flautista, de 61 años, con 47 años como músico y con una media de horas de estudio de 3 por semana durante los últimos 20 ańos, y el músico mayor de la muestra es "un fagot" de 65 años, con 50 ańos de experiencia como instrumentista y con una media entre 3 y 4 horas de estudio.

Dependiendo del tipo de instrumento del músico adulto mayor, la embocadura y los ańos de ejercicio profesional, las fuerzas ejercidas por la lengua, labios, así como las fuerzas de oclusión dental, las del ligamento periodontal y las fuerzas extrínsecas han producido alteraciones en la articulación temporomandibular (ATM) de los músicos adultos.

Entre los flautistas que utilizan una embocadura tipo bisel, la emisión del sonido a lo largo de los años les ha modificado al 100\% la ATM, produciéndose una retrusión mandibular provocada por la forma de la emisión del aire hacia abajo para que se genere el sonido del instrumento, con la consiguiente aparición de dolor en el cuello, rigidez en los músculos mandibulares y "chasquidos" en la mandíbula (fig. 1). En el $75 \%$ se ha producido lo que se conoce como la "barbilla del flautista", que consiste en una descamación e hiperpigmentación del labio inferior, donde existe el contacto con el instrumento.

Todos los clarinetistas que utilizan embocadura de lengüeta simple describen la sensación de cansancio muscular en los labios, sobre todo en el labio inferior, que se conoce como "fatiga crónica de la embocadura", debido a la presión que se ejerce cuando se toca sobre la embocadura del músico. Por la presión ejercida durante tantos años se describe, en el $100 \%$ de la muestra, la aparición de problemas endodónticos que incrementan el overbite o mordida profunda (un tipo de maloclusión que se produce cuando los dientes anteriores inferiores ocluyen por detrás y por dentro de los superiores) como puede observarse en la figura 2 , en el 50\% de los clarinetistas. La utilización de este tipo de embocadura genera en todos ellos lesiones en los tejidos blandos de la cavidad oral, especialmente en el labio inferior, debido a la presión de los incisivos inferiores sobre la boquilla del instrumento.

Entre los oboes y fagotes, instrumentos de embocadura de lengüeta doble, la presión que se genera para producir el sonido ocasiona entre 

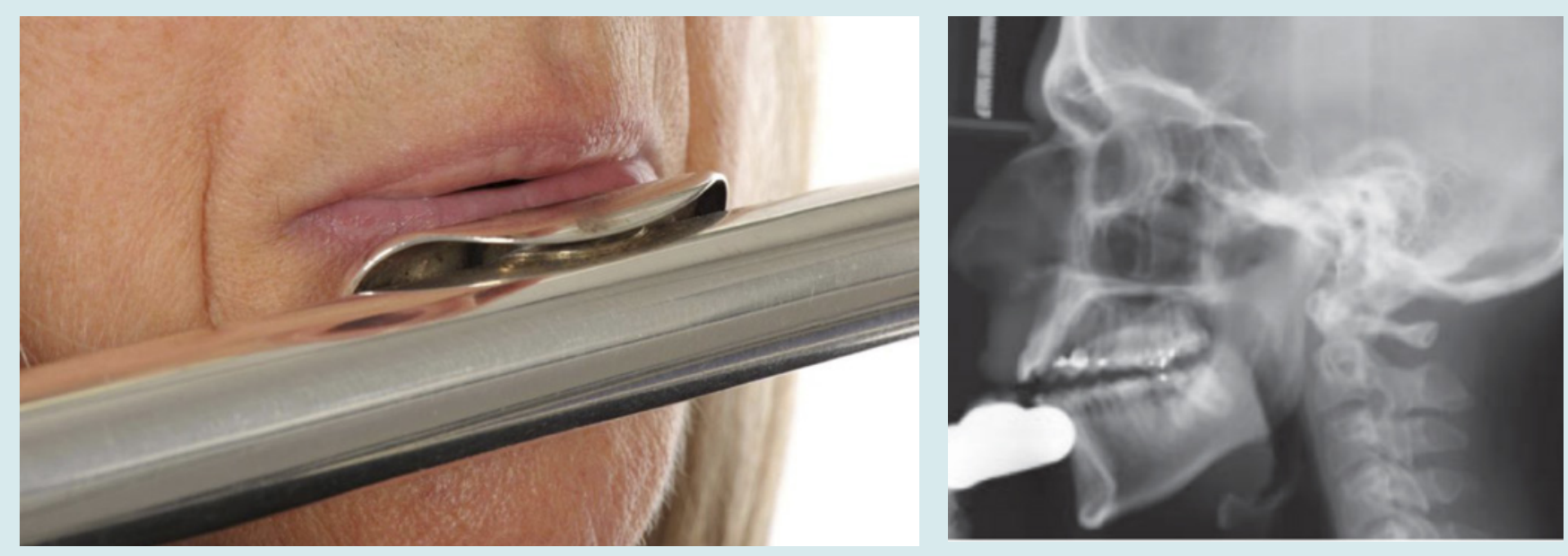

Figura 1. Embocadura tipo Bisel en una flautista adulta mayor.
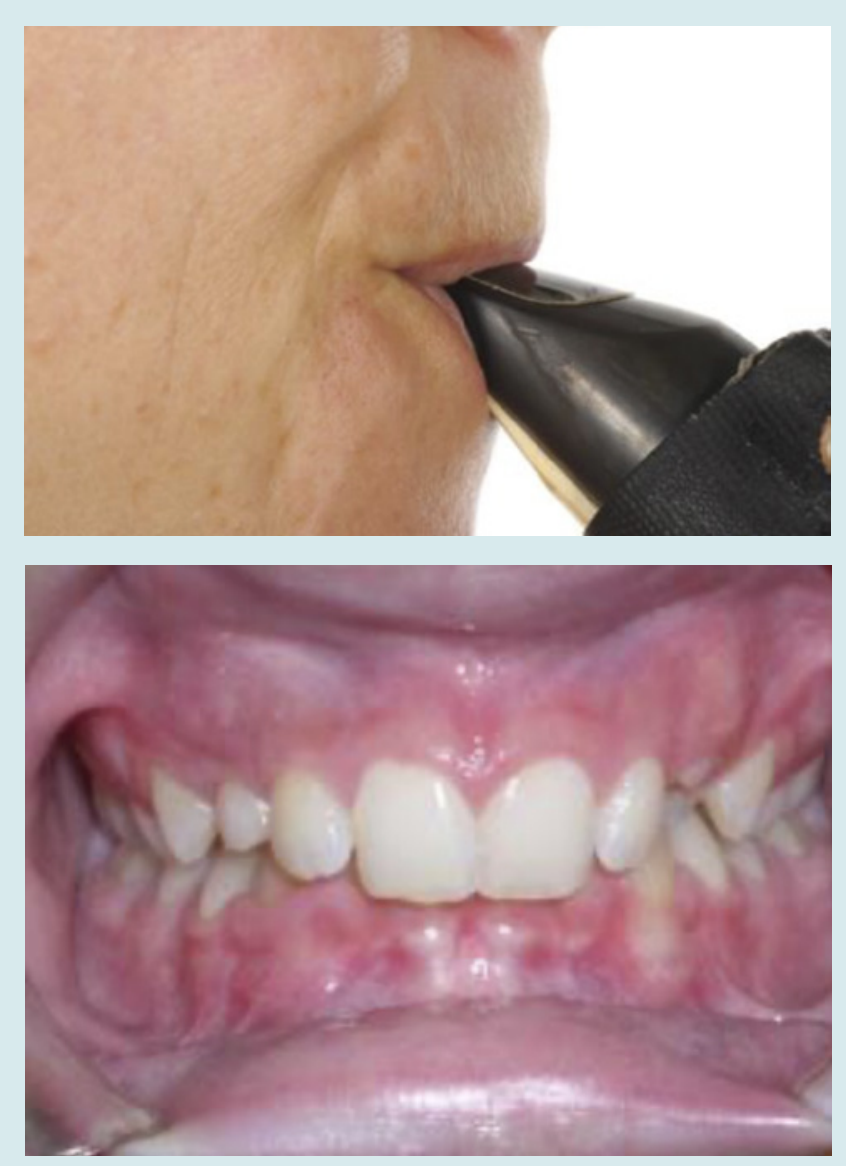

Figura 2. Embocadura simple en músico adulto mayor de clarinete. Maloclusión dental.

todos ellos sintomatología dolorosa a nivel de la ATM, dolor que se irradia a la boca y el cuello, así como lesiones en la mucosa labial, dependiendo del tipo de presión que se ejerza al interpretar una partitura. El 25\% presenta retrognatismo en el aparato estomatognático y el 100\% tiene desgastados los bordes incisales de los incisivos centrales inferiores y superiores.
Es importante resaltar, como factor predisponente en las alteraciones osteoarticulares de la cavidad bucal, la presencia de factores psicosociales. Todos los músicos mayores describen que a lo largo de los años el estrés que padecen durante las horas de estudio y durante los conciertos, así como la biomecánica que utilizan al emitir el sonido, hacen que adopten "posturas antinaturales" (definidas por ellos de esa forma) que les ocasiona molestias a nivel del cuello, cabeza y boca.

Al realizar la comparación con el grupo control, existe diferencia significativa entre la aparición de trastornos de la ATM entre instrumentistas de viento-madera y el grupo control $(p=0,0236)$, donde únicamente hacen referencia a algias a nivel de columna vertebral, normalmente los músicos de instrumentos de percusión, según refieren, debido a la bipedestación mantenida y prolongada durante ensayos y conciertos.

Así mismo, esta significación también se traslada al grupo de los instrumentistas de lengüeta simple y doble, debido a que las maloclusiones que se han detectado entre estos músicos no aparecen entre los percusionistas o pianistas del grupo control $(p=0,0145)$.

\section{- DISCUSIÓN}

Nuestro trabajo demuestra la teoría del movimiento dental según la teoría del equilibrio de Proffit, ya que debido a las fuerzas que ejercen los labios, lengua y todas las estructuras de la boca, acompañadas por las fuerzas extrínsecas capaces de producir en determinados instrumentistas hasta 1000 microdeformaciones al tocar, son suficientes para producir esta movilidad ${ }^{18-19}$.

En esta movilidad y/o deformidad es importante la actuación de las fuerzas de presión de manera intermitente y prolongada, tal como se describe en la tesis doctoral del Instituto Politécnico Nacional de la Escuela Superior de Ingeniería Mecánica y Eléctrica de México ${ }^{20}$, donde analizan la importancia de las fuerzas de presión de forma continuada en el tiempo y de manera intermitente, que se asemeja al mecanismo de estudio que utilizan los músicos durante las horas de estudio. Los resultados de varios estudios realizados con anterioridad al nuestro ${ }^{2-6} \mathrm{se}$ justifican entre los instrumentistas de nuestra investigación con embocadura tipo bisel (flautistas), donde hay que enviar el aire contra el bisel con el fin de producir un sonido tratando de cambiar la velocidad del aire para obtener cambios de registro (fig. 1). Igualmente, tanto los músicos con instrumentos de lengüeta simple (clarinete) como doble (oboe, fagot), para emitir el sonido tienen que expulsar el aire ejerciendo una 
presión determinada para que los músculos de la boca rodeen la boquilla a manera de envoltura, manteniéndose siempre firmes y estables (fig. 2).

Debemos añadir que, tal como defiende Salinas ${ }^{8}$, creemos también que el comenzar a tocar un instrumento desde edades tempranas y mantener la práctica a lo largo de los ańos condiciona la embocadura del músico y la fisiología del aparato estomatognático, efectos que se ha demostrado en nuestros resultados, donde la muestra de nuestro estudio lleva una media de 40,12 $\pm 2,98$ años tocando un instrumento de viento-madera durante una media superior a 3 horas diarias.

Todos estos factores descritos con anterioridad alteran la salud oral, sobre todo en aquellos adultos mayores que se dedican a tocar instrumentos de viento ${ }^{5,8,9}$, tal como se puede comprobar en nuestros resultados, donde se aprecian modificaciones oclusales en los instrumentistas de viento-madera estudiados, maloclusiones que dependiendo del tipo de embocadura pueden tender al retrognatismo mandibular, a un resalte aumentado o por el contrario a una disminución del resalte y aumento de la sobremordida ${ }^{9,11}$.

Además, los músicos del estudio hacen referencia a una sequedad de la cavidad oral durante la interpretación de las partituras, y los oboes y fagotes refieren "desgaste en los dientes incisivos centrales superiores e inferiores", cuadro que coincide con los cambios fisiológicos descritos por Misericordia García y sus colaboradoras ${ }^{16}$ y el equipo de Salgado ${ }^{17}$, aunque se encontró bibliografía donde los investigadores no hallaron efecto entre la tonicidad labial y la posición de los dientes en algunos instrumentistas de viento ${ }^{6}$.

Los efectos que describen los músicos de la muestra debido a situaciones de estrés y factores psicosociales son descritos también en estudiantes jóvenes examinados en centros musicales y orquestas de $\mathrm{México}^{4}$, Chile $^{8}$ y de Europa ${ }^{14}$.

A pesar de que Ortega Velazco, en el tratado Odontoestomatología Geriátrica, plantee que el sexo femenino nota más el cambio de la velocidad del flujo salival, nuestros resultados arrojan valores similares y no significativos en ambos sexos con respecto a la disminución de la secreción salival.

\section{- CONCLUSIONES}

- El presente estudio ha demostrado que tocar un instrumento de viento-madera a nivel profesional durante más de 35 ańos afecta a la posición de los dientes y donde las horas de estudio y las fuerzas continuas e intermitentes necesarias para tocar son factores etiológicos predisponentes de maloclusión.

- El tipo de lengüeta que se utilice para interpretar una partitura y la embocadura influyen a lo largo de los ańos sobre la estructura del aparato estomatognático.

- Las alteraciones osteoarticulares en la cavidad oral de los músicos mayores producen un cuadro clínico que se caracteriza por dolor que se irradia hacia la cara, mandíbula y/o cuello. Puede aparecer también rigidez en los músculos mandibulares, y en ocasiones, con limitaciones del movimiento mandibular.

- En función del tipo de música que se interprete, las alteraciones a nivel de la ATM pueden cambiar y ser dispares.

- La edad, tiempo de dedicación, presión mantenida al tocar el instrumento, así como el tipo de música interpretada, son responsables del desgaste de los dientes, sobre todo en instrumentistas mayores que utilizan lengüeta simple y doble.

- Sería importante reconocer de forma preventiva los signos y síntomas que muestran los instrumentistas adultos mayores durante la interpretación para prevenir los efectos secundarios. Por todo esto, se debería proponer el uso del método Alexander a lo largo de la vida laboral

\section{Conflictos de intereses}

Los autores expresan la inexistencia de conflicto de intereses.

\section{BIBLIOGRAFÍA}

1. Rosinés MD. Músicos y lesiones. Rev Biomecánica. 2010;18(1): 16-8.

2. Lozano Patiño K, Reina Ocampo K, Karime Gómez L, Osori S. Prevalencia de Trastornos Temporomandibulares en Estudiantes de Música. Int J Odontostomat. [Internet]. 2016 [citado 7 de febrero de 2019];10(3):499-505. Disponible en: https://scielo.conicyt.cl/ scielo php?script=sci arttext\&pid=S0718-381X2016000300018\& ng=es. http://dx.doi.org/10.4067/S0718-381X2016000300018

3. Lavado Espíritu JL, Limaymanta Salinas JM. Trastornos Temporomandibulares en Músicos Intérpretes de Instrumentos de Viento Metal - Madera - Huancayo 2017. [Tesis doctoral]. Huancayo-Perú: Universidad Privada de Huancayo "Franklin Roosevelt"; 2017 [acceso 15 de septiembre de 2019]. Disponible en: http://repositorio.uroosevelt edu.pe/xmlui/handle/ROOSEVELT/87

4. Guzmán-Valderrábano CP, Durán-Gutiérrez A, Hernández-Carvallo JR, Valdivia Gómez IG. Instrumentos musicales como factor etiológico de maloclusiones. Rev Mexicana de Ortodoncia. [Internet]. 2018 [citado 9 de marzo de 2019];6(1):35-44. Disponible en: http:// www.medigraphic.com/pdfs/ortodoncia/mo-2018/mo181f.pdf

5. Zwiri A, Al-Omiri M. Prevalence of temporomandibular joint disorder among North Saudi University students. Cranio. 2016;34(3):176-81

6. Pulido MI, Sosa CM, Sosa G. Lesiones del aparato estomatognático en músicos ejecutantes de instrumentos de viento. Estudio de casos. Odous Científica. 2009;10(2):29-38.

7. Van der Weijden FN, Kuitert RB, Berkhout FRU, Van der Weijden $\mathrm{GA}$. Influence of tooth position on wind instrumentalists performance and embouchure comfort: A systematic review. J Orofac Orthop. [Internet]. 2018 [citado 9 de mayo de 2019];79(3):20518. Disponible en: https://www.ncbi.nlm.nih.gov/pmc/articles/ PMC5954010/
8. Salinas JC. Patología funcional del sistema estomatognático en músicos instrumentistas. Rev Hospital Clínico Universidad de Chile [Internet]. 2002 [citado 22 de marzo de 2019]:13(3):171-8. Disponible en: https://es.slideshare.net/joansoco/patologa-funcional-del-sistema-estomatogntico-en-musicos-instrumentistas

9. Rosset i Llobet J. Problemas de embocadura (I). Rev 12 notas. 2004:42:23

10. Blasco Yepes C, Payri B, López Gómez MA. Estudio perceptivo del rebajado de las lengüetas en el oboe. I Congrés Internacional "Investigació en Música": 2010 Febrero 25-26; València, España. València: ISEACV; 2010

11. Duarte Arroyo AN. Relación entre las maloclusiones dentarias y la ejecución de instrumentos de viento madera. [Tesis doctoral]. Caracas, Venezuela: Universidad Central de Venezuela; 2011. [Acceso 2 de febrero de 2019]. Disponible en: https://docplayer es/7102017-Relacion-entre-las-maloclusiones-dentarias-y-la-ejecucion-de-instrumentos-de-viento-madera.html.

12. Montserrat García Gómez M. Las enfermedades profesionales de los músicos, el precio de la perfección. Arch Riesgos Labor. 2018:21(1):11-7.

13. Bluj-Komarnitka K, Komarnitki I, Olczak-Kowalczyk D. Wind Instruments and their Influence on Oral Cavity: Systematic Review. World J Dent. 2014;5(3):180-3

14. Masdevall Galter J Chimenos Küstner E. Los instrumentos musicales como causantes de patología bucal. Rev Europea de Odonto-Estomatología [Internet]. 1998 [citado 11 de diciembre de 2018];10(1):34-8. Disponible en: https://studylib.es/doc/6328172/los-instrumentos-musicales-como-causantes-de-patologia-buca

15. Sáez Carriera R, Carmona M, Jiménez Quintana Z, Alfaro X Cambios bucales en el adulto mayor. Rev Cubana Estomato [Internet]. 2007 Dic [citado 26 de febrero de 2019]:44(4). Dispo- nible en: http://scielo.sld.cu/scielo.php?script=sci_arttext\&pi$d=$ S0034-75072007000400011\&lng=es

16. Noriega Borge MJ, García Hernández M. Torres Egea MP Proceso de Envejecer: Cambios Físicos, Cambios Psíquicos, Cambios Sociales. Capítulo 2. [Internet]. 2005 [citado 26 de febrero de 2019]. Disponible en: http://seegg.es/Documentos/libros/temas/Cap2.pdf

17. Salgado Alba A, Guillén Llera F, Ruipérez I. Manual de Geriatría. 2. ed. Barcelona: Masson; 2002

18. Ortega Velazco D. Envejecimiento oral. En: Bollón Fernández $P$ Velazco Ortega D, eds. Odontoloestomatología geriátrica: la atención odontológica integral del paciente de edad avanzada. Madrid: Coordinación Editorial IMC; 1996. p. 159, 164-6.

19. Morén P. Los instrumentistas de viento ya pueden medir su esfuerzo labial [Internet]. Granada: Universidad de Granada. 2002 [actualizado 15 de mayo de 2002; citado 27 de octubre de 2018]. Disponible en: https://dialogo.ugr.es/contenidos/mayo02/24-iv-tedel.htm

20. Oficina de Mitjans de Comunicació de la UPC. Un equipo de la Escuela Técnica Superior de Ingeniería Industrial de Terrassa, de la UPC, diseña un aparato para tratar enfermedades que afectan a los músicos de viento [Internet]. Barcelona: Universitat Politècnica de Catalunya. 2002 [actualizado 31 de mayo 2002; citado 2 de noviembre de 2018]. Disponible en: https://saladepremsa2.upc.edu/ es/al-dia/mes-noticies/2002/noticia_500-es.html

21. Cerón Monroy I, Sánchez de la Barquera MIC. Acústica Musical. [Tesis doctoral]. Culhuacan. México: Instituto Politécnico Nacional. Escuela Superior de Ingeniería Mecánica y Eléctrica. Unidad Culhuacan. México; 2009. [Acceso 30 de octubre de 2018]. Disponible en: https://tesis.ipn.mx/bitstream/handle/123456789/6898/ ice\%20183.pdf? sequence=1\&isAllowed=y

22. Pacheco del Pino MA. Bandas de Música en los Montes de Toledo: su aportación a la educación musical. [Tesis doctoral]. Valladolid. 
España: Universidad de Valladolid. Departamento de Pedagogía Valladolid; 2012. [Acceso 7 de octubre de 2018]. Disponible en: https://core.ac.uk/download/pdf/61506274.pdf

23. Camelo P. Relatório de prática de ensino supervisionada realizada na Escola de Música do Conservatório Nacional: a embocadura do clarinete: caracterização, deteção e resolucão de problemas. [Trabajo fin de máster-Mestrado em Ensino da Música- en Internet].
Évora. Portugal: Universidade de Évora. Escola de Artes. Departamento de Música; 2016 [acceso 23 de marzo de 2019]. Disponible en: https://dspace.uevora.pt/rdpc/bitstream/10174/20061/1/Relato \%CC $\% 81$ rio $\% 20$ Esta\%CC\%81 gio \%20Patri\%CC $\% 81$ cia $\% 20$ Camelo\%20(Reparado).pdf

24. Gualtieri PA. May Johnny or Janie play the clarinet? The Eastman Study: a report on the orthodontic evaluations of college-level and professional musicians who play brass and woodwind instruments. Am J Orthod. 1979;76(3):260-76

25. Terán $\mathrm{H}$, Castillo A. Efecto del uso de instrumentos de viento en las Maloclusiones dentarias. Revisión de la literatura. Acta Odontológica Venezolana. [Internet]. 2013 [citado 14 de febrero de 2019];51(3). Disponible en: https://www.actaodontologica.com/ ediciones/2013/3/art-17/ 